\title{
THE IRREDUCIBILITY OF CERTAIN PURE-CYCLE HURWITZ SPACES
}

\author{
By Fu LiU and Brian Osserman
}

\begin{abstract}
We study "pure-cycle" Hurwitz spaces, parametrizing covers of the projective line having only one ramified point over each branch point. We start with the case of genus- 0 covers, using a combination of limit linear series theory and group theory to show that these spaces are always irreducible. In the case of four branch points, we also compute the associated Hurwitz numbers. Finally, we give a conditional result in the higher-genus case, requiring at least $3 g$ simply branched points. These results have equivalent formulations in group theory, and in this setting complement results of Conway-Fried-Parker-Völklein.
\end{abstract}

1. Introduction. In this paper, we use a combination of geometric and group-theoretic techniques to prove a result with equivalent statements in both fields. The geometric statement is that certain genus-0 Hurwitz spaces (the "purecycle" cases) are always irreducible, while the group-theoretic statement is that the corresponding factorizations into cycles always lie in a single pure braid group orbit. "Pure-cycle" refers to the hypothesis that for our covers, there is only a single ramified point over each branch point. The main significance for us of this condition is that it allows us to pass relatively freely between the point of view of branched covers, where one moves the branch points freely on the base curve, and linear series, where one moves the ramification points freely on the covering curve. This facilitates induction, as it is easier to stay within the pure-cycle case from the point of view of linear series.

Our result is close to optimal in the sense that if one drops either of the pure-cycle or genus-0 hypotheses, one quickly runs into cases where the Hurwitz spaces have more than one component. However, we do prove a conditional generalization to higher-genus pure-cycle Hurwitz spaces having at least $3 g$ simply branched points, depending on a positive answer to a different geometric question which is closely related to an old question of Zariski.

Our immediate motivation for studying the pure-cycle situation is its relation to linear series: specifically, if one wishes to prove statements on branched covers via linear series arguments, the pure-cycle situation is the natural context to examine. A good understanding of the classical situation is therefore important

Manuscript received October 31, 2006.

Research of the first author supported by fellowships from the Clay Mathematics Institute and MSRI; research of the second author supported in part by a fellowship from the National Science Foundation during the preparation of this paper.

American Journal of Mathematics 130 (2008), 0-00. (C) 2008 by The Johns Hopkins University Press. 
to studying other cases, such as that of positive characteristic. In particular, our main theorem allows for a much simpler proof of a stronger result in [17] than would otherwise be possible, producing sharp Riemann-existence-type results for certain classes of tamely branched branched covers. However, we also remark that a good understanding of the components of Hurwitz spaces has given rise to a wide range of substantial applications: the classical proof of Severi that $\mathcal{M}_{g}$ is connected [11]; number-theoretic applications such as Davenport's problem, and Thompson's genus-0 problems in group theory, both due to Fried [5]; and the Fried-Völklein description of the absolute Galois group of certain fields in inverse Galois theory [9], [10]. As such, our results have significant potential for applications in the direction of inverse Galois theory. Already, our explicit results in the case of 4 branch points have been used by Fried in [8] to verify his Main Conjecture for modular towers in an infinite family of new examples; see the end of $\S 5$ below for a brief synopsis.

We now state our results more precisely. We will recall/fix our terminology in the next section.

The following proposition is well known, although the equivalence of the first two and last two conditions depends heavily on the fact that we restrict our attention to covers with a single ramified point over each branch point. We will recall the proof in the following section.

Proposition 1.1. Given $d$ and $\vec{e}=\left(e_{1}, \ldots, e_{r}\right)$ with $2 d-2=\sum_{i}\left(e_{i}-1\right)$, the following are equivalent:

(a) the Hurwitz factorizations for $(d, r, 0, \vec{e})$ all lie in a single orbit of the pure braid group.

(b) the space $\mathcal{H}(d, r, 0, \vec{e})$ is irreducible, where $\mathcal{H}(d, r, 0, \vec{e})$ is the Hurwitz space parametrizing $r$ distinct points $Q_{1}, \ldots, Q_{r}$ on $\mathbb{P}^{1}$ together with a genus-O cover of $\mathbb{P}^{1}$, such that each $Q_{i}$ has a single point over it ramified to order $e_{i}$, and the rest unramified;

(c) the space $M R:=\operatorname{MR}\left(\mathbb{P}^{1}, \mathbb{P}^{1}, \vec{e}\right)$ is irreducible, where $M R$ is the space parametrizing $r$ distinct points $P_{1}, \ldots, P_{r}$ on $\mathbb{P}^{1}$ together with a rational function $f: \mathbb{P}^{1} \rightarrow \mathbb{P}^{1}$ of degree $d$ and ramified to order $e_{i}$ at $P_{i}$ (on the source curve) for all $i$;

(d) the space $G_{d}^{1}:=G_{d}^{1}\left(\mathbb{P}^{1}, \vec{e}\right)$ is irreducible, where $G_{d}^{1}$ is the space parametrizing $r$ distinct points $P_{1}, \ldots, P_{r}$ on $\mathbb{P}^{1}$ together with a linear series of dimension 1 and degree $d$, having ramification $e_{i}$ at $P_{i}$ for all $i$;

Our main theorem is then the following:

THEOREM 1.2. Given $d$, and $e_{1}, \ldots, e_{r}$ with $2 d-2=\sum_{i}\left(e_{i}-1\right)$, the equivalent conditions of Proposition 1.1 always hold.

Our proof follows the general structure of Eisenbud and Harris' argument in [3], where they prove the irreducibility of certain families of linear series without prescribed ramification. However, while they work exclusively from the 
perspective of linear series, we have to switch back and forth between points of view. Starting from the perspective of linear series, we use a degeneration argument and the tools of limit linear series to reduce to a base case of four points on $\mathbb{P}^{1}$, and then solve that case directly, after switching to the grouptheoretic point of view of Hurwitz factorizations. Our explicit work in the base case also computes the Hurwitz numbers for that case:

THEOREM 1.3. Given $d$ and $\vec{e}=\left(e_{1}, \ldots, e_{4}\right)$ with $2 d-2=\sum_{i}\left(e_{i}-1\right)$, we have the following formula for the Hurwitz number:

$$
h(d, r, 0, \vec{e})=\min \left\{e_{i}\left(d+1-e_{i}\right)\right\}_{i} .
$$

We can also describe the Hurwitz factorizations in this case completely explicitly.

In Theorem 5.5 below, we again use limit linear series techniques to prove a conditional version of Theorem 1.2 for pure-cycle cases of higher genus having at least $3 g$ simply branched points, depending on a positive answer to Question 5.4 below, a geometric question closely related to an old question of Zariski.

We conclude by arguing that the combination of the genus- 0 and pure-cycle conditions imply that our monodromy groups are always either cyclic, $S_{d}$, or $A_{d}$; we show this, independently of the proof of our main results, in Theorem 5.3 below.

Our higher-genus result could be seen as having the spirit of an effective version in the pure-cycle case of results of Conway-Fried-Parker-Völklein [9, Appendix]. Our main theorem also generalizes a theorem of Fried [7, Thm. 1.2], which implies the case of our Theorem 1.2 in which $e_{i}=3$ for all $i$. Finally, Hurwitz spaces of polynomial covers were considered by Khovanskii and Zdravkovska in [13], where in particular they recovered our result in the case of polynomial pure-cycle covers.

Acknowledgments. We would like to thank Michael Fried, Kay Magaard and David Harbater for help with context and references, and Robert Guralnick for assistance with the proof of Theorem 5.3.

2. Notation and terminology. We quickly recall terminology and fix our notation. For geometric statements, we assume throughout that we are working over $\mathbb{C}$.

Our notation for permutations will always be to express them as products of cycles. Given $\sigma \in S_{d}$, we will say that a number $k \in\{1, \ldots, d\}$ is in the support of $\sigma$ if $\sigma(k) \neq k$.

Given a permutation $\sigma$ (or conjugacy class $T$ ) of $S_{d}$, we define its index $\iota(\sigma)$ as follows: if $a_{1} \leq a_{2} \leq \ldots \leq a_{m}$ is the corresponding partition, then $\iota(\sigma):=\sum_{i=1}^{m}\left(a_{i}-1\right)$. We then say that a tuple $\left(d, r, g,\left(T_{1}, \ldots, T_{r}\right)\right)$ constitutes 
the data of a Hurwitz problem, where $d \geq 1, r \geq 2, g \geq 0$, the $T_{i}$ are conjugacy classes in $S_{d}$, and we require $2 d-2+2 g=\sum_{i} \iota\left(T_{i}\right)$.

Associated to a Hurwitz problem we have the group-theoretic question of finding all Hurwitz factorizations $\left(\sigma_{1}, \ldots, \sigma_{r}\right)$, where:

(i) $\sigma_{i} \in T_{i}$;

(ii) $\sigma_{1} \ldots \sigma_{r}=1$;

(iii) the $\sigma_{i}$ generate a transitive subgroup of $S_{d}$.

We say that two Hurwitz factorizations are equivalent if they are related by simultaneous conjugation by an element of $S_{d}$. We call the number of equivalence classes of Hurwitz factorizations the Hurwitz number $h\left(d, r, g,\left(T_{1}, \ldots, T_{r}\right)\right)$. See Remark 2.2 below for discussion of some relating and conflicting notation in the literature.

Geometrically, we also have the Hurwitz space $\mathcal{H}\left(d, r, g,\left(T_{1}, \ldots, T_{r}\right)\right)$, parametrizing $r$-tuples of marked points on $\mathbb{P}^{1}$, together with covers of degree $d$ and genus $g$, unramified away from the marked points, and with monodromy type $T_{i}$ at the $i$ th marked point for all $i$. For a fixed choice of marked points, such covers correspond to Hurwitz factorizations up to equivalence, so the degree of $\mathcal{H}\left(d, r, g,\left(T_{1}, \ldots, T_{r}\right)\right)$ over the space $\mathcal{M}_{0, r}$ parametrizing marked points is given by the Hurwitz number.

We say that a Hurwitz problem is pure-cycle if each $T_{i}$ consists of a single cycle. Throughout this paper, we restrict our attention to pure-cycle Hurwitz problems, and we replace the $T_{i}$ by integers $e_{i} \geq 2$ giving the length of the cycle. We thus have $2 d-2+2 g=\sum_{i}\left(e_{i}-1\right)$ as the condition on our data.

We recall that the Artin braid group $B_{r}$ acts on tuples $\left(\sigma_{1}, \ldots, \sigma_{r}\right)$ in $S_{d}$ with $\sigma_{1} \ldots \sigma_{r}=1$, preserving the group generated by the $\sigma_{i}$. The $i$ th generator acts by replacing $\left(\sigma_{i}, \sigma_{i+1}\right)$ by $\left(\sigma_{i+1}, \sigma_{i+1}^{-1} \sigma_{i} \sigma_{i+1}\right)$. The kernel of the natural map $B_{r} \rightarrow S_{r}$ is the pure braid group, which not only preserves $\sigma_{1} \ldots \sigma_{r}=1$, but sends each $\sigma_{i}$ to a conjugate of itself in the group generated by all the $\sigma_{i}$. We thus see that the pure braid group acts on the set of Hurwitz factorizations, and it is the orbits of this action which we will study.

Note as a consequence of the geometric definition of Hurwitz number that the number is clearly invariant under reordering of the $e_{i}$. We can also see this purely in terms of group theory by making use of the braid group action to permute the $e_{i}$ arbitrarily.

We will also be working from the point of view of linear series, which from our point of view will always have dimension 1 and be basepoint free: in this situation, a linear series of dimension 1 and degree $d$ (also called a $\mathfrak{g}_{d}^{1}$ ) on a curve $C$ is simply a map to $\mathbb{P}^{1}$ of degree $d$, considered up to automorphism of the image space. We remark that the basepoint-free hypothesis will not cause us any problems, as we will always be working with spaces of linear series with all ramification specified.

As a simple case of the sort of analysis we will carry out in the four-point case, we recall the answer in the case of three points: 
Lemma 2.1. The Hurwitz number for $\left(d, 3,0,\left(e_{1}, e_{2}, e_{3}\right)\right)$ is always 1 , corresponding to the factorization:

$$
\begin{aligned}
& \sigma_{1}=\left(d-e_{2}, d-e_{2}-1, \ldots, 2,1, e_{3}, e_{3}+1, e_{3}+2, \ldots, d-1, d\right), \\
& \sigma_{2}=\left(d, d-1, \ldots, d-e_{2}+2, d-e_{2}+1\right), \text { and } \\
& \sigma_{3}=\left(1,2, \ldots, e_{3}-1, e_{3}\right) .
\end{aligned}
$$

Proof. First, note that by transitivity and the fact that $\sigma_{1}=\sigma_{3}^{-1} \sigma_{2}^{-1}$, we have that $\sigma_{3}$ and $\sigma_{2}$ together act nontrivially on all of $\{1, \ldots, d\}$, and their actions must therefore overlap on a subset of cardinality exactly $e_{2}+e_{3}-d=d+1-e_{1}$.

To complete the proof, one observes that if a sequence of precisely $m$ consecutive elements in the cycle representation of $\sigma_{2}$ also appear in $\sigma_{3}$, at most $m-1$ of them can remain fixed by $\sigma_{2} \sigma_{3}$. It follows that in order for $\sigma_{2} \sigma_{3}$ to be an $e_{1}$-cycle, the overlap must form a single contiguous portion of each of $\sigma_{2}$ and $\sigma_{3}$, from which one easily concludes the desired statement.

Finally, we recall:

Proof of Proposition 1.1. The equivalence of (i) and (ii) is classical and quite general: the basic idea is that the monodromy cycles of a cover depend not only on the cover, but also on a choice of local monodromy generators of the fundamental group of the base; all such choices of generators are related by braid operations, and each braid operation can be achieved as monodromy of the Hurwitz space by moving the marked points of the base around one another. For a slightly different exposition, see [20, Prop. 10.14 (a)]; note that the situation is slightly different because he considers Hurwitz spaces with unordered branch points and full braid orbits, but the argument is the same in our case of ordered branch points and pure braid orbits.

Similarly, the equivalence of (iii) and (iv) is equally basic: the space $G_{d}^{1}$ is obtained from the space $M R$ simply by modding out by the (free) action of the automorphism group of the base $\mathbb{P}^{1}$, so $M R$ is a $P G L_{2}$-bundle over $G_{d}^{1}$, and one space is irreducible if and only if the other is.

Next, because we have restricted to Hurwitz spaces in which there is a single ramified point over each branch point, the comparison of $M R$ and $\mathcal{H}(d, r, 0, \vec{e})$ is almost equally straightforward. First suppose $r \geq 3$. If we denote by $\widehat{M R}$ the open subscheme of $M R$ for which the map $f$ sends the marked ramification points to distinct points, then because $r \geq 3$, we have that $\widehat{M R}$ is a $P G L_{2}$-bundle over $\mathcal{H}(d, r, 0, \vec{e})$, so one is irreducible if and only if the other is. But then an easy deformation-theory argument shows that any component of $M R$ dominates the $\left(\mathbb{P}^{1}\right)^{r}$ parametrizing the branch points of the map $f$ [15, Cor. 3.2], so we see that $\widehat{M R}$ is dense in $M R$, completing the desired equivalences for irreducibility. Finally, if $r=2$, the only maps are, up to automorphism, $x \mapsto x^{d}$, so it is easy to see that both $M R$ and $\mathcal{H}(d, r, 0, \vec{e})$ are irreducible. 
Remark 2.2. Our terminology of Hurwitz problem (and more specifically, the associated set of Hurwitz factorizations) is closely related to the more standard terminology "Nielsen class", for which one also specifies a subgroup $G$ which the $\sigma_{i}$ must generate, and assigns the $T_{i}$ as conjugacy classes within that subgroup.

The Nielsen class is frequently better because it gives a finer combinatorial invariant: the Hurwitz factorizations for a given Hurwitz problem are a disjoint union over different Nielsen classes, and likewise the Hurwitz space is a disjoint union over spaces associated to different Nielsen classes. Our main theorem immediately implies that for the cases we study, a Hurwitz problem consists of only a single Neilsen class. See also Theorem 5.3 below for a direct proof of this fact.

One has to be slightly careful in comparing statements, since the Nielsen class terminology also allows for different equivalence relations on the Hurwitz factorizations (for instance, working up to inner automorphism of $G$ ).

We also remark on our terminology of Hurwitz number. First, in [4] the term "Hurwitz number" is used to describe the number of components of the Hurwitz space, while what we call the Hurwitz number is called the "degree". Second, in many other papers, a "stacky" definition of Hurwitz number is used in which rather than working up to equivalence, one counts all Hurwitz factorizations, and divides by $d$ !. This is the same as counting covers divided by the number of their automorphisms, and reflects the fact that in general, a Hurwitz space might be a stack with nontrivial stabilizer groups. However, in the pure-cycle genus0 case, this will not affect the answer as long as $r \geq 3$, since one can check using Theorem 5.3 below that $S_{d}$ always acts without fixed points on the set of Hurwitz factorizations, which implies that our covers are automorphism-free and the Hurwitz space is in fact a scheme.

3. Reduction to four points. The goal of this section is to use the machinery of limit linear series to prove:

PROPOSITION 3.1. To prove Theorem 1.2 in general, it is enough to give a proof in the case that $r=4$.

In order to use a degeneration argument for Proposition 3.1, the key fact which we need (and which is lacking in the higher-genus case) is:

Proposition 3.2. Every component of the space $G_{d}^{1}$ of Proposition 1.1 maps dominantly under the forgetful map to $\mathcal{M}_{0, r}$.

Proof. Indeed, we know [1, Thm. 2.3] that if we fix ramification points, we have only finitely many $\mathfrak{g}_{d}^{1}$, s with the prescribed ramification, and that conversely, if we move the branch points, our rational function can always be deformed [15, Cor. 3.2]; the statement then follows by a dimension count, as in the proof of ibid. 
We will make essential use of the $r=1$ case of limit linear series, developed by Eisenbud and Harris in [2]. We briefly review the critical points of their theory in this case, where it becomes considerably simpler. Suppose that we have a family $\mathcal{C}$ of curves, with smooth generic fiber, but with some nodal fibers. We assume that all nodal fibers are of compact type, i.e., that their dual graph is a tree. Eisenbud and Harris construct a space over all of $\mathcal{C}$ which correspond to usual $\mathfrak{g}_{d}^{1}$ 's on smooth fibers of $\mathcal{C}$, but correspond to limit linear series on the nodal fibers; by abuse of notation, we write $\mathfrak{g}_{d}^{1}$ to mean also limit linear series. Suppose that $C$ is a nodal fiber with (necessarily smooth) components $C_{1}, \ldots, C_{m}$. In our case of $r=1$, a (refined) limit linear series on $C$ may be expressed as an $m$-tuple of aspects on each $C_{i}$, where an aspect is a $\mathfrak{g}_{d_{i}}^{1}$ with $d_{i} \leq d$, and the sole compatibility condition is that if $C_{i}$ and $C_{j}$ meet at a node $P$, then the ramification index at $P$ of the aspects on $C_{i}$ and $C_{j}$ should be the same. Given $r$ smooth sections $P_{i}$ of $\mathcal{C}$, the Eisenbud-Harris construction also works to give spaces of $\mathfrak{g}_{d}^{1}$,s with at least a specified amount of ramification at the $P_{i}$ (in fact, limit linear series should in general allow for base points away from the nodes, but since we will work with the case that all ramification is specified, this won't arise).

We review the situation further in the case $g=0$, with all ramification specified. This is studied in [14, Thm. 2.4]; there, the families considered involve only breaking off one component at a time, but our assertions here easily follow by the same arguments. For the rest of the section, we fix our degenerate curve:

Situation 3.3. The curve $C_{0}$ is the totally degenerate curve given by a nodal chain of $r-2$ copies of $\mathbb{P}^{1}$, with $P_{1}, P_{2}$ on the first component, $P_{i}$ on the $(i-1)$ st component for $i<2<r-1$, and $P_{r-1}, P_{r}$ on the last component.

We consider families $\mathcal{C}$ near a fiber isomorphic to the specified $C_{0}$. Because all ramification is specified, the space of $\mathfrak{g}_{d}^{1}$ 's is finite over $\mathcal{C}$, and is in fact finite étale in a neighborhood of $C_{0}$. Furthermore, a $\mathfrak{g}_{d}^{1}$ on $C_{0}$ is uniquely described by a collection of ramification indices $\left(e_{2}^{\prime}, \ldots, e_{r-2}^{\prime}\right)$ at the nodes, which are required to satisfy a collection of triangle inequalities and a parity condition. Specifically, if we consider any consecutive triple $e, e^{\prime}, e^{\prime \prime}$ starting with an odd-indexed term in the sequence

$$
e_{1}, e_{2}, e_{2}^{\prime}, e_{3}, \ldots, e_{r-2}, e_{r-2}^{\prime}, e_{r-1}, e_{r}
$$

we need to have $e \leq e^{\prime}+e^{\prime \prime}, e^{\prime} \leq e+e^{\prime \prime}$, and $e^{\prime \prime} \leq e+e^{\prime}$, and we need $e+e^{\prime}+e^{\prime \prime}$ to be odd.

For later use, we note that the second condition implies immediately that the triangle inequalities are in fact always strict, and also that the allowed parity of $e_{2}^{\prime}, \ldots, e_{r-2}^{\prime}$ is fixed by the $e_{i}$.

With these tools in hand, we can now complete our geometric argument. 
Proof of Proposition 3.1. We fix the totally degenerate curve $C_{0}$ as in the above situation, and work with a local universal family $\mathcal{C}$ of genus- 0 curves in a neighborhood of $C_{0}$, denoting the generic curve of this family (which is also the generic curve of $\mathcal{M}_{0, r}$ ) by $C_{\eta}$. It is enough to show that the relative $G_{d}^{1}$ space (with the desired ramification at the marked points) is irreducible over the family $\mathcal{C}$, since by the previous proposition, every component of the global $G_{d}^{1}$ space meets the generic curve $C_{\eta}$. By the same token, it is enough to show that any two $\mathfrak{g}_{d}^{1}$ 's on the geometric generic fiber $\bar{C}_{\eta}$ lie on the same component of $G_{d}^{1}$. Furthermore, because the space of $\mathfrak{g}_{d}^{1}$ 's is reduced over $C_{0}$, we have cannot have two components of $G_{d}^{1}$ meet over $C_{0}$, so over our family $\mathcal{C}$, irreducible components of $G_{d}^{1}$ are the same as connected components.

Accordingly, suppose we are given two $\mathfrak{g}_{d}^{1}$,s on $\bar{C}_{\eta}$. By the above discussion, these can be specialized to $\mathfrak{g}_{d}^{1}$ 's on $C_{0}$, which are described by the data of ramification indices $\left(e_{2}^{\prime}, \ldots, e_{r-2}^{\prime}\right)$ and $\left(e_{2}^{\prime \prime}, \ldots, e_{r-2}^{\prime \prime}\right)$ respectively. We set the convention that $e_{1}^{\prime}=e_{1}^{\prime \prime}:=e_{1}$, and $e_{r-1}^{\prime}=e_{r-1}^{\prime \prime}:=e_{r}$. Our claim is as follows: if we assume the $r=4$ case of Theorem 1.2 , then any two $\mathfrak{g}_{d}^{1}$ 's on $C_{0}$ such that $e_{i}^{\prime}=e_{i}^{\prime \prime}$ for all but one $i$ necessarily lie on the same component of $G_{d}^{1}$.

Indeed, if we fix a node of $C_{0}$ corresponding to $e_{i}^{\prime}$ (i.e., the $(i-1)$ st node), we can restrict the family $\mathcal{C}$ to the closed subfamily $\mathcal{C}_{i}$ in which only the chosen node of $C_{0}$ is allowed to be smoothed, giving a smooth component containing the two marked points $P_{i}$ and $P_{i+1}$, and the $(i-2)$ nd and $i$ th nodes (unless $i=2$ or $r-2$, in which case $P_{1}$ or $P_{r}$ takes the place of the $(i-2)$ nd or $i$ th node respectively). The other components remain fixed, so we may consider $\mathcal{C}_{i}$ to be obtained from the universal family over $\overline{\mathcal{M}}_{0,4}$ by localizing around a degenerate curve, and gluing appropriate chains of $\mathbb{P}^{1}$,s at the first and fourth marked points; in particular, the base of $\mathcal{C}_{i}$ is naturally a local scheme $U$ of $\overline{\mathcal{M}}_{0,4}$ at a point corresponding to a degenerate curve. If we write $\mathcal{C}_{0,4}$ for the universal curve over $U$, the point is to relate the $G_{d}^{1}$ spaces associated to $\mathcal{C}_{0,4}$ and $\mathcal{C}_{i}$.

Specifically, suppose we have chosen indices $e_{j}^{\prime}=e_{j}^{\prime \prime}$ for all $j \neq i$. For the sake of clarity, we denote by $G_{d}^{1}(\mathcal{C})$ our original space of $\mathfrak{g}_{d}^{1}$, s on $\mathcal{C}$, and by $G_{d}^{1}\left(\mathcal{C}_{i}\right)$ and $G_{d}^{1}\left(\mathcal{C}_{0,4}\right)$ the spaces of $\mathfrak{g}_{d}^{1}$,s on $\mathcal{C}_{i}$ and $\mathcal{C}_{0,4}$. For the first two spaces, we impose ramification $e_{i}$ at each $P_{i}$, so that $G_{d}^{1}\left(\mathcal{C}_{i}\right)$ is simply the base change of $G_{d}^{1}(\mathcal{C})$, while for $G_{d}^{1}\left(\mathcal{C}_{0,4}\right)$ we impose ramification $e_{i-1}^{\prime}, e_{i}, e_{i+1}, e_{i+1}^{\prime}$ at the four marked points. Now, if we consider the closed subscheme $Z_{i}$ of $G_{d}^{1}\left(\mathcal{C}_{i}\right)$ which corresponds to limit $\mathfrak{g}_{d}^{1}$, s with ramification indices $e_{j}^{\prime}$ at the nodes (for $j \neq i$ ), the limit $\mathfrak{g}_{d}^{1}$ 's are uniquely determined except on the component with four marked points, so $Z_{i}$ is isomorphic to the space $G_{d}^{1}\left(\mathcal{C}_{0,4}\right)$ which we have described. Thus if we assume Theorem 1.2 in the case $r=4$, we see that the subscheme $Z_{i}$ of $G_{d}^{1}\left(\mathcal{C}_{i}\right)$ is irreducible, so that any two $\mathfrak{g}_{d}^{1}$ 's on $C_{0}$ for which $e_{i}^{\prime}=e_{i}^{\prime \prime}$ for all but one $i$ lie on the same connected component of $G_{d}^{1}\left(\mathcal{C}_{i}\right)$, and hence of $G_{d}^{1}(\mathcal{C})$.

This proves the claim, and since every limit $\mathfrak{g}_{d}^{1}$ on $C_{0}$ can be smoothed to a $\mathfrak{g}_{d}^{1}$ on $\bar{C}_{\eta}$, the following numerical lemma completes the proof of our proposition. 
LEMMA 3.4. Let $C_{0}$ be a totally degenerate marked curve of genus 0 , and suppose we are given two $\mathfrak{g}_{d}^{1}$ 's with ramification indices $e_{i}$ at the marked points, and classified by ramification indices $\left(e_{2}^{\prime}, \ldots, e_{r-2}^{\prime}\right)$ and $\left(e_{2}^{\prime \prime}, \ldots, e_{r-2}^{\prime \prime}\right)$ respectively at the nodes. Then it is possible to modify $\left(e_{2}^{\prime}, \ldots, e_{r-2}^{\prime}\right)$ into $\left(e_{2}^{\prime \prime}, \ldots, e_{r-2}^{\prime \prime}\right)$, by a sequence of changes affecting only one index at a time, and with every intermediate set of indices corresponding to a valid $\mathfrak{g}_{d}^{1}$ on $C_{0}$.

Proof. Suppose we have a $\mathfrak{g}_{d}^{1}$ on $C_{0}$ specified by the set $\left(e_{2}^{\prime}, \ldots, e_{r-2}^{\prime}\right)$. Since the allowed parity of each of $e_{2}^{\prime}, \ldots, e_{r-2}^{\prime}$ is fixed by the $e_{i}$, as long as we change them by 2 at a time, we do not need to worry about violating the parity condition. It is thus enough to show that if $\left(e_{2}^{\prime}, \ldots, e_{r-2}^{\prime}\right)$ and $\left(e_{2}^{\prime \prime}, \ldots, e_{r-2}^{\prime \prime}\right)$ are distinct, there is always some $i$ with $e_{i}^{\prime} \neq e_{i}^{\prime \prime}$ and for which we can increase $e_{i}^{\prime}$ or $e_{i}^{\prime \prime}$ to make it closer to the other without violating any triangle inequalities. We prove this by induction.

We will induct on the following statement: suppose we are given $i$ such that $e_{i}^{\prime \prime}-e_{i}^{\prime} \geq e_{i-1}^{\prime \prime}-e_{i-1}^{\prime}$ and $e_{i}^{\prime}+2 \leq e_{i-1}^{\prime}+e_{i}$. Then either we can increase $e_{i}^{\prime}$, or we must have $e_{i+1}^{\prime \prime}-e_{i+1}^{\prime} \geq e_{i}^{\prime \prime}-e_{i}^{\prime}$ and $e_{i+1}^{\prime}+2 \leq e_{i}^{\prime}+e_{i+1}$. Indeed, if we cannot increase $e_{i}^{\prime}$, the only triangle inequalities that could be violated are $e_{i}^{\prime}+2 \leq e_{i-1}^{\prime}+e_{i}$ or $e_{i}^{\prime}+2 \leq e_{i+1}+e_{i+1}^{\prime}$. But the first one is satisfied by hypothesis, so the only possibility is that $e_{i}^{\prime}+2>e_{i+1}+e_{i+1}^{\prime}$, in which case we see we must have $e_{i}^{\prime}+1=e_{i+1}+e_{i+1}^{\prime}$. But we then see that

$$
e_{i+1}^{\prime \prime}-e_{i+1}^{\prime}=e_{i+1}^{\prime \prime}+e_{i+1}-e_{i}^{\prime}-1 \geq e_{i}^{\prime \prime}-e_{i}^{\prime}
$$

by the triangle inequality. Furthermore, $e_{i+1}^{\prime}+2 \leq e_{i}^{\prime}+e_{i+1}$ because $e_{i+1} \geq 2$.

Now suppose that $i_{0}$ is the smallest number with $e_{i_{0}}^{\prime} \neq e_{i_{0}}^{\prime \prime}$. Without loss of generality, we may assume that $e_{i_{0}}^{\prime}<e_{i_{0}}^{\prime \prime}$. But we see that this satisfies the hypotheses of our inductive statement: the first inequality is clear since $e_{i_{0}-1}^{\prime \prime}=$ $e_{i_{0}-1}^{\prime}$, while the second follows because we have $e_{i_{0}}^{\prime}+2 \leq e_{i_{0}}^{\prime \prime} \leq e_{i_{0}-1}^{\prime \prime}+e_{i_{0}}=$ $e_{i_{0}-1}^{\prime}+e_{i_{0}}$. But by induction, we see that we must eventually be able to increment one of the $e_{i}^{\prime}$ for $i \geq i_{0}$, since when $i=r-2$, we have $e_{r-1}^{\prime \prime}=e_{r-1}^{\prime}=e_{r}$. This proves the lemma.

4. The case of four points. In this section, we study the case of four points from the group-theoretic point of view. Our setup throughout this section is as follows:

Situation 4.1. We are given $d>0$, and $\vec{e}:=\left(e_{1}, e_{2}, e_{3}, e_{4}\right)$, with $2 d-2=$ $\sum_{i}\left(e_{i}-1\right)$, and $2 \leq e_{1} \leq e_{2} \leq e_{3} \leq e_{4} \leq d$.

We observe for later use that in our situation, we have $e_{1}+e_{3} \leq d+1$, $e_{2}+e_{4} \geq d+1, e_{1}+e_{2} \leq d+1$, and $e_{3}+e_{4} \geq d+1$. The first two inequalities follow from $e_{1}+e_{3} \leq e_{2}+e_{4}$ together with $e_{1}+e_{2}+e_{3}+e_{4}=2 d+2$, while the second two follow by comparing with the first two. 
Throughout this section, we will write sequences of the form $i, i+1, \ldots, j$ (and similarly for descending sequences). If $j \geq i$, the meaning is clear: an ascending sequence of length $j-i+1$. However, without further comment we will also allow $j=i-1$, in which case the meaning will be the empty sequence (still of length $j-i+1)$.

Our main result is the following:

THeOREM 4.2. In Situation 4.1, the Hurwitz number $h(d, 4,0, \vec{e})$ is given by $\min \left\{e_{i}\left(d+1-e_{i}\right)\right\}_{i}$.

Moreover, the possible Hurwitz factorizations $\left(\sigma_{1}, \sigma_{2}, \sigma_{3}, \sigma_{4}\right)$ are classified explicitly as follows:

(i) if $\sigma_{3} \sigma_{4}$ is trivial or a single cycle, then we have

$$
\begin{aligned}
\sigma_{1}= & \left(d, d-1, \ldots, e_{3}+e_{4}+1-k,\right. \\
& \left.\sigma^{-\left(d+2-k-e_{1}\right)}(\ell), \sigma^{-\left(d+3-k-e_{1}\right)}(\ell), \ldots, \sigma^{-\left(e_{3}+e_{4}+1-2 k\right)}(\ell)=\ell\right), \\
\sigma_{2}= & \left(e_{3}+e_{4}+1-k, e_{3}+e_{4}+2-k, \ldots, d-1, d, \ell, \sigma^{-1}(\ell), \ldots, \sigma^{-\left(d+1-k-e_{1}\right)}(\ell)\right), \\
\sigma_{3}= & \left(k, k-1, \ldots, 2,1, e_{4}+1, e_{4}+2, \ldots, e_{3}+e_{4}-k\right), \\
\sigma_{4}= & \left(1, \ldots, e_{4}\right),
\end{aligned}
$$

where we allow any $k$ with $e_{3}+e_{4}-d \leq k \leq e_{3}$ and $k \leq d+1-e_{2}$, we define $\sigma:=\left(k, k+1, \ldots, e_{3}+e_{4}-k\right)=\sigma_{3} \sigma_{4}$, and for a given $k$, we allow $\ell$ to vary in the range $k \leq \ell \leq e_{3}+e_{4}-k$.

(ii) if $\sigma_{3} \sigma_{4}$ is a product of two disjoint cycles, then we have

$$
\begin{aligned}
\sigma_{1}= & \left(m+e_{1}-1, m+e_{1}-2, \ldots, m+1, m\right), \\
\sigma_{2}= & \left(d, d-1, \ldots, m+e_{1}, m+d+k-e_{3}-e_{4}, m+d-1+k-e_{3}-e_{4}, \ldots, k\right), \\
\sigma_{3}= & \left(k, k-1, \ldots, 1, e_{4}+1, e_{4}+2, \ldots, m+e_{1}-1,\right. \\
& \left.\quad m, m-1, \ldots, m+d+1+k-e_{3}-e_{4}, m+e_{1}, m+e_{1}+1, \ldots, d\right), \\
\sigma_{4}= & \left(1, \ldots, e_{4}\right),
\end{aligned}
$$

where we allow any $k$ with $1 \leq k \leq e_{3}+e_{4}-d-1$, and any $m$ with $e_{4}-e_{1}+1 \leq$ $m \leq d+1-e_{1}$ and $m \leq e_{4}$.

Before giving the proof, we give a number of simple technical lemmas and their consequences; although each result individually is quite easy and presumably well-known, we include them for the sake of staying as self-contained as possible.

We begin by simplifying the transitivity condition on Hurwitz factorizations in our situation.

Lemma 4.3. Suppose that $\sigma_{1}, \sigma_{2}, \sigma_{3}, \sigma_{4} \in S_{d}$ are cycles of length $e_{1}, e_{2}, e_{3}, e_{4}$, with trivial product. Then the following are equivalent:

(a) the $\sigma_{i}$ form a Hurwitz factorization for $(d, 4,0, \vec{e})$;

(b) every number in $\{1, \ldots, d\}$ is in the support of at least one of the $\sigma_{i}$; 
(c) every number in $\{1, \ldots, d\}$ is in the support of exactly two of the $\sigma_{i}$, except that either there is some $k$ which is in the support of every $\sigma_{i}$, or there exist $k \neq \ell$, with each in the support of three of the $\sigma_{i}$.

Proof. It is clear that the transitivity condition for (a) implies (b). To see that (b) implies (c), the key point is that the identity $2 d-2=\sum_{i}\left(e_{i}-1\right)$ leaves relatively little flexibility for the $\sigma_{i}$. Specifically, if every number in $\{1, \ldots, d\}$ is in the support of at least one cycle, it must be in the support of at least two, as otherwise the product could not be trivial. But we have $\sum_{i} e_{i}=2 d+2$, and $2 d$ of the numbers in the support of the $\sigma_{i}$ are accounted for, leaving only 2 which could be in the support of more than two cycles. We conclude (c).

Finally, to see that (c) implies (a), we need only check transitivity. Since every number is in the support of at least two $\sigma_{i}$, we cannot have any $\sigma_{i}$ disjoint from all the others. Thus, the only way they could fail to generate a transitive subgroup would be if two of the $\sigma_{i}$ were disjoint from the other two. But this cannot occur, as the inequalities $e_{3}+e_{4} \geq d+1$ and $e_{2}+e_{4} \geq d+1$ imply that $\sigma_{4}$ cannot be disjoint from either of $\sigma_{2}$ or $\sigma_{3}$.

We next pursue a detailed study of the relationship between pairs of cycles and their products.

Lemma 4.4. Suppose $\sigma_{1}, \sigma_{2} \in S_{d}$ are nondisjoint cycles in $S_{d}$, and let $\sigma$ be any cycle in the decomposition of $\sigma_{1} \sigma_{2}$ into disjoint cycles. Then there exists an element of $\{1, \ldots, d\}$ in the support of $\sigma, \sigma_{1}$, and $\sigma_{2}$.

Proof. This is routine: if $\sigma$ consisted entirely of numbers in the support of $\sigma_{1}$ but not $\sigma_{2}$, it would have to be equal to $\sigma_{1}$, contradicting the nondisjointness hypothesis, and similarly with the $\sigma_{i}$ reversed. One then verifies that to switch from elements in the support of $\sigma_{1}$ to elements in the support of $\sigma_{2}$ requires an element of $\sigma$ in the support of both.

Lemma 4.5. Let $\sigma, \sigma^{\prime}$ be nondisjoint cycles, with $\sigma \sigma^{\prime} \neq 1$. Then there exists $a$ unique expression (up to cycling of indices) of $\sigma^{\prime}$ as $\left(w_{1}^{\prime}, v_{1}^{\prime}, \ldots, w_{m}^{\prime}, v_{m}^{\prime}\right)$ and $\sigma$ as $\left(w_{1}, v_{1}, w_{2}, v_{2}, \ldots, w_{m}, v_{m}\right)$ where the $w_{i}^{\prime}, v_{i}^{\prime}$ and $w_{i}, v_{i}$ are sequences of numbers, satisfying:

(i) the $w_{i}$ and $w_{i}^{\prime}$ are all nonempty, but the $v_{i}$ and $v_{i}^{\prime}$ may be empty;

(ii) each $v_{i}^{\prime}$ consists of numbers not in the support of $\sigma$;

(iii) each $v_{i}$ consists of numbers not in the support of $\sigma^{\prime}$;

(iv) there exists a permutation $\tau \in S_{m}$ such that each $w_{i}$ is the inverse of $w_{\tau(i)}^{\prime}$ (i.e., the same sequence in reversed order);

(v) iffor all $i$ we set $k_{i}$ to be the first number in $w_{i}$, the set of $k_{i}$ is precisely the set of numbers in the support of all three of $\sigma, \sigma^{\prime}$, and $\sigma \sigma^{\prime}$.

Proof. By Lemma 4.4, there is some number in the support of $\sigma$, of $\sigma^{\prime}$ and of $\sigma \sigma^{\prime}$; we begin by designating one such number to be $k_{1}$. In order to be able 
to write $\sigma$ in the desired form, the order of the remaining $k_{i}$ are then uniquely determined. Furthermore, we see that each $w_{i}$ must consist of the longest word in $\sigma$ which starts with $k_{i}$, contains only numbers also in the support of $\sigma^{\prime}$, and does not contain $k_{j}$ for $j \neq i$. This uniquely determines each $w_{i}$, and the $v_{i}$ are what remain. We can then do the same for the $w_{i}^{\prime}$ and $v_{i}^{\prime}$, except that the $k_{i}$ could appear in a different order in $\sigma^{\prime}$, giving us the permutation $\tau$. It remains to check that these expressions have the desired properties, specifically (ii), (iii), and (iv).

Note that if $n \neq k_{i}$ for any $i$ is any number in the support of $\sigma$ and $\sigma^{\prime}$, since $n$ isn't in the support of $\sigma \sigma^{\prime}$, then $\sigma^{\prime}(n)=\sigma^{-1}(n)$, so we see that $\sigma^{\prime}(n)$ must also be in the support of $\sigma$, immediately prior to $n$ in the cycle representation. Applying this inductively gives that all such $n$ appear in the $w_{i}$ in $\sigma$ and in the $w_{i}^{\prime}$ in $\sigma^{\prime}$, and that each $w_{\tau(i)}^{\prime}$ is inverse to $w_{i}$, as desired.

The following corollary is quite special to the case of at most two repetitions.

COROLlary 4.6. Let $\sigma, \sigma^{\prime}$ be cycles, and write $S \subseteq\{1, \ldots, d\}$ for the intersection of the supports of $\sigma, \sigma^{\prime}$ and $\sigma \sigma^{\prime}$. Suppose that either:

(I) $\sigma=\sigma_{3}, \sigma^{\prime}=\sigma_{4},\left(\sigma_{1}, \sigma_{2}, \sigma_{3}, \sigma_{4}\right)$ a Hurwitz factorization for $(d, 4,0, \vec{e})$;

(II) $\sigma$ and $\sigma^{\prime}$ are not disjoint, and $\# S \leq 2$.

Then the number of disjoint cycles in $\sigma \sigma^{\prime}$ is equal to \#S and is at most 2, and there is exactly one element of $S$ in the support of each disjoint cycle.

Proof. We first observe that (I) implies (II). Indeed, $\sigma_{3}$ and $\sigma_{4}$ cannot be disjoint since $e_{3}+e_{4} \geq d+1$, and we have $\# S \leq 2$ by Lemma 4.3(c), since any $k$ which occurs in the support of $\sigma_{3} \sigma_{4}$ must also occur in the support of $\sigma_{1}$ or $\sigma_{2}$ because of the trivial-product condition.

We next argue that (II) implies the conclusion of the corollary. Lemma 4.4 immediately handles the case $\# S \leq 1$. For $\# S=2$, we apply the above lemma, noting first that in this case we can always cycle indices so that $\tau=1$, i.e., each $w_{i}^{\prime}$ is the inverse of $w_{i}$. Then note that the formula

$$
\left(w_{1}, v_{1}, w_{2}, v_{2}\right)\left(w_{1}^{\prime}, v_{1}^{\prime}, w_{2}^{\prime}, v_{2}^{\prime}\right)=\left(k_{1}, v_{1}^{\prime}, v_{2}\right)\left(k_{2}, v_{2}^{\prime}, v_{1}\right)
$$

holds regardless of whether the $v_{i}$ and $v_{i}^{\prime}$ have nonzero length; since $k_{1}$ and $k_{2}$ are assumed to be in the support of $\sigma \sigma^{\prime}$, we see that it must consist of two disjoint cycles.

We are now ready to give the proof of Theorem 4.2. For the sake of clarity, we break the proof into four propositions.

Proposition 4.7. Each of the possibilities enumerated in Theorem 4.2 gives a valid Hurwitz factorization, and $\sigma_{3} \sigma_{4}$ is in the asserted form (and in particular, consists of the asserted number of cycles).

Proof. The main technicality is to check that the individual cycles themselves make sense. This involves checking three points: first, that all the elements listed lie in $\{1, \ldots, d\}$; second, that each word has nonnegative length; and third, that 
there is no overlap between the words in a given cycle. In fact, we first see that all words have nonnegative length, which then simplifies checking that their ranges are appropriate.

Before that, we observe that $\sigma=\left(k, k+1, \ldots, e_{3}+e_{4}-k\right)$ makes sense: the length is at least 1 , since we have $2 k \leq 2 e_{3} \leq e_{3}+e_{4}$; and we check both $k \geq 1$ and $e_{3}+e_{4}-k \leq d$ using the inequality $e_{3}+e_{4}-d \leq k$ (together with $e_{3}+e_{4} \geq d+1$ for the first).

In general, we allow sequences to have length 0 , except those containing $k$, $\ell$, or $m$. In case (i), for $\sigma_{1}$ we require that $d \geq e_{3}+e_{4}-k$ and $e_{3}+e_{4}+1-2 k \geq$ $d+2-k-e_{1}$, which are equivalent to $k \geq e_{3}+e_{4}-d$ and $k \leq d+1-e_{2}$ respectively. For $\sigma_{2}$, we need $e_{3}+e_{4}-k \leq d$ and $d+1-k-e_{1} \geq 0$, giving $k \geq e_{3}+e_{4}-d$ and $k \leq d+1-e_{1}$ respectively. Since $d+1-e_{2} \leq d+1-e_{1}$, the last inequality will also be satisfied. Finally, for $\sigma_{3}$ we need $k \geq 1$ and $e_{3}+e_{4}-k \geq e_{4}$; the first is satisfied since $e_{3}+e_{4}-d \geq 1$, while the second is simply $k \leq e_{3}$. Case (ii) is similar, with the only inequality appearing other than those imposed directly being $m \geq e_{3}+e_{4}-d$. However, this is okay, since we have $m \geq e_{4}-e_{1}+1$, and the inequality $e_{1}+e_{3} \leq d+1$ implies that $e_{3}+e_{4}-d \leq e_{4}-e_{1}+1$. Thus, the ranges provided guarantee that the cycles make sense, and are in fact equivalent to having the lengths of all words containing $k, \ell$, or $m$ be at least 1 , and the lengths of the remaining words being at least 0 .

We next address the first and third points simultaneously. In case (i), everything follows easily from the ranges imposed for $k$ : for $\sigma_{4}$ there is nothing to check; for $\sigma_{3}$ we check that $k<e_{4}+1$ and $e_{3}+e_{4}-k \leq d$; and for $\sigma_{2}$ and $\sigma_{1}$, everything is immediate, since the terms involving $\sigma$ are automatically in the correct range, and the sequence in $\sigma_{1}$ involving $\sigma$ could not wrap around without the sequence in $\sigma_{2}$ having negative length, and vice versa.

Similarly, in case (ii), the only points requiring any nonimmediate checking are: for $\sigma_{3}$, that $k<m+d+1+k-e_{3}-e_{4}$, and $m<e_{4}+1$, with the former following from $e_{1}+e_{3} \leq d+1$; for $\sigma_{2}$, that $m+d+k-e_{3}-e_{4}<m+e_{1}$; and for $\sigma_{1}$, that $m \geq 1$. Thus, all the cycles consist of nonoverlapping entries in $\{1, \ldots, d\}$.

We can then check directly that the cycles are of the correct length and have trivial product, as well as that $\sigma=\sigma_{3} \sigma_{4}$. Finally, using that b) implies a) in Lemma 4.3 makes it easy to check that the cycles generate transitive subgroups of $S_{d}$, so all the possibilities listed are valid Hurwitz factorizations.

It remains only to note that in case (i), we have already written $\sigma$ explicitly, so we see that $\sigma_{3} \sigma_{4}$ is in fact trivial or a single cycle, while in case (ii), we check that $m>m+d+k-e_{3}-e_{4}$, so that $\sigma_{1}$ is disjoint from $\sigma_{2}$, and since $\sigma_{1} \sigma_{2} \sigma_{3} \sigma_{4}=1$, it follows that $\sigma_{3} \sigma_{4}$ is a product of two disjoint cycles.

Proposition 4.8. No two possibilities enumerated in Theorem 4.2 are equivalent.

Proof. Cases (i) and (ii) of Theorem 4.2 are clearly invariant under relabeling. In case (i), we see that $k$ is determined as the number of elements in the support of 
both $\sigma_{3}$ and $\sigma_{4}$, so is invariant under relabeling. If $\sigma=1$ (i.e., if $k=e_{3}+e_{4}-k$ ), we have $\ell=k$ is the only possibility. Given $k$ with $\sigma \neq 1$, we see that $\ell$ is determined as the unique number (in the allowed range) such that $\sigma^{\ell-k}(k)$ is in the support of $\sigma_{1}$ and $\sigma_{2}$ (and necessarily $\sigma$ ), so two possibilities with different $\ell$ cannot be equivalent.

In case (ii), the size of the intersection of the supports of $\sigma_{1}$ and $\sigma_{4}$ is $e_{4}+1-m$, so $m$ is relabeling-invariant. The overlap between the supports of $\sigma_{3}$ and $\sigma_{4}$ consists of two contiguous words, and $k$ is determined as the length of the word with nonempty overlap with $\sigma_{2}$. Hence, no two possibilities are equivalent.

Proposition 4.9. Every Hurwitz factorization is equivalent to one of the possibilities enumerated in Theorem 4.2.

Proof. We begin by noting that by Corollary 4.6, we must have that $\sigma_{3} \sigma_{4}$ consists of 0,1 , or 2 disjoint cycles. Furthermore, if $\sigma_{3} \sigma_{4}=1$, then we have $\sigma_{3}=\sigma_{4}^{-1}$, and $\sigma_{1}=\sigma_{2}^{-1}$, and $e_{1}=e_{2}=d+1-e_{3}=d+1-e_{4}$, so it is easy to check that the only possibility is the $k=\ell=e_{3}=e_{4}$ case of (i). We can thus assume that $\sigma_{3} \sigma_{4} \neq 1$.

The first case we consider is that $\sigma=\sigma_{3} \sigma_{4}$ is a single cycle, or, equivalently by Corollary 4.6, that there is a single number $k^{\prime} \in\{1, \ldots, d\}$ which is in the support of $\sigma_{3}, \sigma_{4}$, and in $\sigma=\sigma_{3} \sigma_{4}$. Let $k$ be the number of elements in the support of both $\sigma_{3}$ and $\sigma_{4}$. We may then relabel so that $\sigma_{4}=\left(1, \ldots, e_{4}\right)$, and $k^{\prime}$ gets mapped to $k$; i.e, so that the unique number in the support of $\sigma_{3}, \sigma_{4}$, and $\sigma$ is $k$. Applying Lemma 4.5 to $\sigma_{3}$ and $\sigma_{4}$ with the only $k_{i}$ being $k$ gives us that $\sigma_{3}$ is necessarily of the form $\left(k, k-1, \ldots, 2,1, a_{1}, \ldots, a_{e_{3}-k}\right)$ for some $a_{i} \in\left\{e_{4}+1, \ldots, d\right\}$; relabeling the latter range allows us to put $\sigma_{3}$ in the desired form.

Next, note that by Lemma 4.3, there must be a unique number $\ell$ in the support of $\sigma_{1}$, of $\sigma_{2}$, and of $\sigma$. We then have also by Lemma 4.3 that all the numbers $\left\{e_{3}+e_{4}-k+1, \ldots, d\right\}$ must be in the support of $\sigma_{2}$, and we claim that they must be in a contiguous word, and followed immediately by $\ell, \sigma^{-1}(\ell), \ldots, \sigma^{-\left(d+1-k-e_{1}\right)}(\ell)$. The claim is checked by applying Lemma 4.5 to $\sigma_{2}$ and $\sigma$, using that $\sigma_{2} \sigma=\sigma_{1}^{-1}$, so that the only $k_{i}$ is $k_{1}=\ell$. The claim implies that we are free to reorder $\left\{e_{3}+e_{4}-k+1, \ldots, d\right\}$ so that they appear in order, and furthermore so that $\sigma_{2}(d)=\ell$. Hence, we have put $\sigma_{2}$ in the desired form, and then $\sigma_{1}$ is determined by $\sigma_{1} \sigma_{2} \sigma=1$.

We next consider the case that $\sigma$ is a product of two disjoint cycles, which by Corollary 4.6 is equivalent to having two numbers $k^{\prime}, k^{\prime \prime} \in\{1, \ldots, d\}$ which are each in the support of $\sigma_{3}, \sigma_{4}$, and in $\sigma:=\sigma_{3} \sigma_{4}$. Then $k^{\prime}$ is in one of the disjoint cycles of $\sigma$, and $k^{\prime \prime}$ is in the other. By Lemma 4.3, we see that since we already have $k^{\prime}, k^{\prime \prime}$ occurring in $\sigma_{3}, \sigma_{4}$ and $\sigma$ (hence in either $\sigma_{1}$ or $\sigma_{2}$ ), we cannot have any numbers occurring in $\sigma_{1}, \sigma_{2}$ and $\sigma$. By Corollary 4.6 (II), we see that $\sigma_{1}$ and $\sigma_{2}$ must be disjoint, and since $\sigma_{1} \sigma_{2}=\sigma^{-1}$, we see that $k^{\prime}$ is in the support of one, 
and $k^{\prime \prime}$ is in the support of the other; without loss of generality, we may assume that $k^{\prime}$ is in the support of $\sigma_{1}$ and $k^{\prime \prime}$ in $\sigma_{2}$. We also note that this implies that each of $\{1, \ldots, d\}$ is in the support of either $\sigma_{3}$ or $\sigma_{4}$.

We once again normalize so that $\sigma_{4}=\left(1, \ldots, e_{4}\right)$, and we can further require that if we write $\sigma_{3}=\left(w_{1}, v_{1}, w_{2}, v_{2}\right)$ and $\sigma_{4}=\left(w_{1}^{\prime}, v_{1}^{\prime}, w_{2}^{\prime}, v_{2}^{\prime}\right)$ as in Lemma 4.5, we can set $w_{1}^{\prime}=(1,2, \ldots, k)$, with $k$ being the corresponding relabeling of $k^{\prime \prime}$, i.e., the unique number in the support of $\sigma_{3}, \sigma_{4}$, and $\sigma_{2}$. We then have $w_{1}$ in the desired form, and $w_{2}$ will likewise be in the desired form for some $m$, which will necessarily be the unique number in the support of $\sigma_{3}, \sigma_{4}$, and $\sigma_{1}$. Relabelling $e_{4}+1, \ldots, d$ as necessary, we can place $v_{1}$ and $v_{2}$, hence $\sigma_{3}$ in the desired form, and $\sigma_{1}$ and $\sigma_{2}$ are then uniquely determined as disjoint cycles with $\sigma_{1} \sigma_{2} \sigma=1$, and containing $m$ and $k$ respectively.

This then completes the proof of the claim that every Hurwitz factorization is equivalent to one of the enumerated possibilities.

Proposition 4.10. The number of possibilities enumerated in Theorem 4.2 is equal to $\min \left\{e_{i}\left(d+1-e_{i}\right)\right\}_{i}$.

Proof. The formula $\min \left\{e_{i}\left(d+1-e_{i}\right)\right\}_{i}$ falls into two situations: if $e_{4} \geq$ $d+1-e_{1}$, then it is equal to $e_{4}\left(d+1-e_{4}\right)$, while if $e_{4} \leq d+1-e_{1}$, then it gives $e_{1}\left(d+1-e_{1}\right)$.

We first consider the situation that $e_{4} \geq d+1-e_{1}$. Here, because $e_{4}+e_{1} \geq d+1$, we have $e_{2}+e_{3} \leq d+1$, so $e_{3} \leq d+1-e_{2}$, and in case (i) of Theorem 4.2 the inequality $e_{3}+e_{4}-d \leq k \leq e_{3}$ automatically implies $k \leq d+1-e_{2}$. We thus have

$$
\sum_{k=e_{3}+e_{4}-d}^{e_{3}} \sum_{\ell=k}^{e_{3}+e_{4}-k} 1=\sum_{k=e_{3}+e_{4}-d}^{e_{3}}\left(e_{3}+e_{4}-2 k+1\right)=\left(d+1-e_{3}\right)\left(d+1-e_{4}\right)
$$

possibilities from case (i). Similarly, we have $d+1-e_{1} \leq e_{4}$ so $e_{4}-e_{1}+1 \leq$ $m \leq d+1-e_{1}$ implies that $m \leq e_{4}$. Thus, our ranges are $1 \leq k \leq e_{3}+e_{4}-d-1$ and $e_{4}-e_{1}+1 \leq m \leq d+1-e_{1}$, yielding $\left(e_{3}+e_{4}-d-1\right)\left(d+1-e_{4}\right)$ possibilities in case (ii), and giving us the desired $e_{4}\left(d+1-e_{4}\right)$ possibilities in total (note that $e_{3}+e_{4}-d-1$ and $d+1-e_{4}$ are always nonnegative, so these formulas are always valid).

The situation that $e_{4} \leq d+1-e_{1}$ proceeds similarly, with $e_{1} e_{2}$ possibilities arising from case (i), and $e_{1}\left(d+1-e_{1}-e_{2}\right)$ possibilities arising from case (ii).

Combining the statements of the four propositions, we immediately conclude Theorem 4.2.

From the theorem, we deduce quite directly:

CoRollary 4.11. In Situation 4.1, the Hurwitz factorizations for $(d, 4,0, \vec{e})$ all lie in a single pure braid orbit. 
Proof. We first see that all the factorizations in case (i) of the theorem are in a single pure braid orbit, and then show that any factorization in case (ii) is in the same braid orbit as some factorization in case (i).

Suppose we start with $\left(\sigma_{1}, \sigma_{2}, \sigma_{3}, \sigma_{4}\right)$ corresponding to a given $k, \ell$ of case (i). Our first claim is that if we replace $\left(\sigma_{1}, \sigma_{2}\right)$ by $\left(\sigma_{2}^{-1} \sigma_{1} \sigma_{2}, \sigma_{2}^{-1} \sigma_{1}^{-1} \sigma_{2} \sigma_{1} \sigma_{2}=\right.$ $\sigma \sigma_{2} \sigma^{-1}$ ), we stay in case (i), leaving $k$ fixed, while replacing $\ell$ by $\sigma(\ell)$. The first part is clear, while the assertion on $\ell$ is checked by direct computation, using that since $\sigma_{3}, \sigma_{4}$ remain fixed, it is enough to see what happens to $\sigma_{2}$. Thus, for a given $k$, every possible $\ell$ is in the same braid orbit.

To analyze the Hurwitz factorizations for different $k$, for each $k$ we set $\ell=k$, where we have $\sigma_{1}=\left(d, d-1, \ldots, e_{3}+e_{4}+1-k, d+1-e_{2}, d-e_{2}, \ldots, k\right)$ and hence

$$
\begin{aligned}
\sigma_{2} \sigma_{3}=\sigma_{1}^{-1} \sigma_{4}^{-1}= & \left(k, k-1, \ldots, 1, e_{4}, e_{4}-1, \ldots, d+2-e_{2},\right. \\
& \left.e_{3}+e_{4}+1-k, e_{3}+e_{4}+2-k, \ldots, d\right) .
\end{aligned}
$$

We check that if we replace $\left(\sigma_{2}, \sigma_{3}\right)$ by $\left(\sigma_{2}^{\prime}, \sigma_{3}^{\prime}\right):=\left(\sigma_{3}^{-1} \sigma_{2} \sigma_{3}, \sigma_{3}^{-1} \sigma_{2}^{-1} \sigma_{3} \sigma_{2} \sigma_{3}\right)$, then as long as $k$ is not minimal, we remain in case (i), but replace $k$ by $k-1$. Here, a relabeling is in principle necessary, but we can instead check that $\sigma_{3}^{\prime} \sigma_{4}$ is still a single cycle, so that we remain in case (i), and that the supports of $\sigma_{3}^{\prime}$ and $\sigma_{4}$ overlap in $k-1$ elements. We therefore see that every possibility in (i) is always in a single pure braid orbit.

Finally, we suppose we have $\left(\sigma_{1}, \sigma_{2}, \sigma_{3}, \sigma_{4}\right)$ corresponding to a given $k, m$ of case (ii). In this case, we again replace $\left(\sigma_{2}, \sigma_{3}\right)$ by $\left(\sigma_{2}^{\prime}=\sigma_{3}^{-1} \sigma_{2} \sigma_{3}, \sigma_{3}^{\prime}=\right.$ $\left.\sigma_{3}^{-1} \sigma_{2}^{-1} \sigma_{3} \sigma_{2} \sigma_{3}\right)$, and note that since $\sigma_{1}, \sigma_{4}$ remain unchanged, $\sigma_{2}^{\prime}$ determines $\sigma_{3}^{\prime}$. One then computes that as long as $k<e_{3}+e_{4}-d-1, \sigma_{2}^{\prime}$ is still a possibility from case (ii), with $m$ the same, but $k+1$ instead of $k$. Finally, if $k=e_{3}+e_{4}-d-1$, one checks that applying the same pure braid operation, we move into case (i), with $k=e_{3}+e_{4}+d$ (and $\ell=m$ ). Thus, every possibility in case (ii) is in the same pure braid orbit as some possibility in case (i), and we get that everything is in the same pure braid orbit.

Using Proposition 1.1, and Proposition 3.1, we see immediately that Corollary 4.11 implies Theorem 1.2, and we are done.

5. Loose ends. We begin with a further remark in the case of four points. The Hurwitz number $\min \left\{e_{i}\left(d+1-e_{i}\right)\right\}_{i}$ computes the number of rational functions $\mathbb{P}^{1} \rightarrow \mathbb{P}^{1}$ with four fixed branch points on the target. If instead we look at fixed ramification points on the source, we find that the number is $\min \left\{e_{i}, d+1-e_{i}\right\}_{i}[14$, Rem. 5.9]. Despite the close geometric relationship between these two numbers, there is no a priori reason for there to be any numerical relationship at all, so their similarity is striking. We note further that with the exception of the case 
that we have $e_{i}=d$ for some $d$, both formulas are symmetric with respect to replacing the $e_{i}$ by $d+1-e_{i}$; this motivates us to ask:

Question 5.1. Is there a natural involution on the set of rational functions of degree $d$ having exactly four ramification points, which replaces the ramification indices $e_{i}$ by $d+1-e_{i}$, and holds both the ramification and branch points fixed?

A more obvious question left unanswered by our analysis is:

Question 5.2. Is there a closed form for the genus-0 pure-cycle Hurwitz numbers for any number of branch points?

Next, we observe that it is a consequence of Theorem 1.2 that if we fix $d, r$ and $\vec{e}$, all possible Hurwitz factorizations are in a single Nielsen class, i.e., they generate the same group, and are in the same conjugacy classes within that group. However, with some nontrivial group theory and sufficient perseverance, one can already see this quite directly:

THEOREM 5.3. Given $d, r$ and $\vec{e}=\left(e_{1}, \ldots, e_{r}\right)$ with $2 d-2=\sum_{i}\left(e_{i}-1\right)$, and all $e_{i} \geq 2$, suppose we have $\left(\sigma_{1}, \ldots, \sigma_{r}\right)$ and $\left(\sigma_{1}^{\prime}, \ldots, \sigma_{r}^{\prime}\right)$ two Hurwitz factorizations for $(d, r, 0, \vec{e})$, generating groups $G, G^{\prime} \subseteq S_{d}$. Then there exists a simultaneous conjugation in $S_{d}$ making $G^{\prime}=G$, and each $\sigma_{i}$ conjugate to $\sigma_{i}^{\prime}$ inside $G$. That is, any two Hurwitz factorizations lie in the same Nielsen class.

In fact, if $r=2$, we have $G$ isomorphic to the cyclic group $C_{d}$. If $r=3$ with $\left(e_{1}, e_{2}, e_{3}\right)=(4,4,5)$, we have $G \cong S_{5}$, imbedded as a doubly transitive subgroup of $S_{6}$. Otherwise, we always have $G=S_{d}$ or $G=A_{d}$ depending on the parity of the $e_{i}$.

Proof. The case that $r=2$ is clear, as we must have $e_{1}=e_{2}=d$.

For $r=3$, we note that the first assertion is clear, since the Hurwitz number is equal to 1 by Lemma 2.1 .

For $r>3$, we reduce the first assertion to the second. In the case that $G=S_{d}$, this is trivial, while in the case that $G=A_{d}$, we need only observe that since $2 d-2=\sum_{i}\left(e_{i}-1\right)$, and all $e_{i} \geq 3$, we can have at most one cycle of order greater than $d-2$. We can always fix this cycle by simultaneous conjugation in $S_{d}$, and then any cycles of given length less than or equal to $d-2$ are in the same conjugacy class in $A_{d}$.

For the second assertion, we begin by arguing that with $r>2$, we must have $G$ primitive, i.e., that there is no nontrivial partition of $\{1, \ldots, d\}$ into blocks on which the action of $G$ is well-defined. Indeed, if there were such a partition, since $G$ is transitive the blocks would all have to have the same size $m$, for some $m \mid d$. We would then necessarily have each $\sigma_{i}$ either of size a multiple of $m$, acting as a $e_{i}^{\prime}:=\frac{e_{i}}{m}$-cycle $\sigma_{i}^{\prime}$ on $d^{\prime}:=\frac{d}{m}$ blocks of size $m$, or of size strictly less than $m$, acting trivially on the blocks. Say we have $s$ of the latter; without loss of generality, we may assume that $e_{1}, \ldots, e_{s}<m$, and $e_{s+1}, \ldots, e_{r} \geq m$. Then $\sigma_{s+1}^{\prime}, \ldots, \sigma_{r}^{\prime}$ give a Hurwitz factorization in $S_{d^{\prime}}$, so we must have $2 d^{\prime}-2 \leq \sum_{i=s+1}^{r}\left(e_{i}^{\prime}-1\right)$. On the 
other hand, we compute that since $2 d-2=\sum_{i}\left(e_{i}-1\right)$, we have $2 d+r-2=\sum_{i} e_{i}$, so

$$
2 \frac{d}{m}+\frac{r-2}{m}-\sum_{i=1}^{s} \frac{e_{i}}{m}=\sum_{i=s+1}^{r} \frac{e_{i}}{m}=\sum_{i=s+1}^{r} e_{i}^{\prime},
$$

and so $2 d^{\prime}-2-\delta=\sum_{i=s+1}^{r}\left(e_{i}^{\prime}-1\right)$, where

$\delta=\sum_{i=1}^{s} \frac{e_{i}}{m}-s+r-2-\frac{r-2}{m} \geq \frac{2 s}{m}-s+r-2-\frac{r-2}{m}=\frac{(m-1)(r-s-2)}{m}+\frac{s}{m}$,

so we must have $\frac{(m-1)(r-s-2)}{m}+\frac{s}{m} \leq 0$. Since the $\sigma_{i}^{\prime}$ act transitively on $d^{\prime}$ elements, and have trivial product, there must be at least 2 of them which are nontrivial, so that $r-s-2 \geq 0$. Since $m>1$, we see that $\delta \geq 0$, and we can have $\delta=0$ only if $r-s-2=s=0$, i.e., $r=2$. Thus, with our hypothesis that $r>2$, we must have $\delta>0$, a contradiction.

We note that in the case that $d \leq 3$, the only transitive subgroups are $A_{d}$ and $S_{d}$, so there is nothing to prove. In the case $d=4$, one checks directly that there is no primitive subgroup other than $S_{4}$ and $A_{4}$, so we need only consider the case $d \geq 5$.

Now, we wish to apply the theorem of Williamson [21] stating that if a primitive subgroup of $S_{d}$ contains a cycle of order $e$, with $e \leq(d-e)$ !, then it must be either $A_{d}$ or $S_{d}$. Since we have $2 d-2=\sum_{i}\left(e_{i}-1\right)$, we see that we must have $e_{i} \leq\left\lfloor\frac{2 d-2}{r}+1\right\rfloor$ for some $i$. One then computes directly that Williamson's theorem gives the desired result unless we have $r=3, d \leq 10$, or $r=4, d \leq 5$. More specifically, the only cases falling outside Williamson's theorem are $r=3$ with $\left(e_{1}, e_{2}, e_{3}\right)=(3,4,4),(4,4,5),(5,5,5),(7,7,7)$ or $r=4$ with $\left(e_{1}, e_{2}, e_{3}, e_{4}\right)=(3,3,3,3)$. In these cases, one can check directly that the group is $A_{d}$ or $S_{d}$, as appropriate, except in the $(4,4,5)$ case, where one can compute the group explicitly, checking that it is doubly transitive and has order 120 , which is well-known to determine it uniquely.

Our result is sharp in the sense that if one drops either the pure-cycle or the genus-0 hypothesis, there are many examples for which the Hurwitz space is not irreducible. However, there are nonetheless many examples for which the Hurwitz space is irreducible which are not covered by our main theorem. We will consider here one generalization which remains in the pure-cycle case, but seeks to drop the genus- 0 hypothesis in favor of an assumption that could be viewed philosophically as an effective form of the results of Conway-FriedParker-Völklein, in that it requires at least $3 g$ transpositions in order to apply. However, our result will be conditional on a positive answer to a geometric question, which we now discuss. 
Zariski asked whether every Hurwitz space of genus- $g$ covers of $\mathbb{P}^{1}$ with prescribed branching type over at least $3 g$ points maps dominantly to $\mathcal{M}_{g}$ under the forgetful map. This is now known to be false in some cases, but we will be interested in an analogous yet different question which arises when one wants to compare the points of view of linear series and branched covers:

Question 5.4. Fix $r, g \geq 0, d \geq 1$ and $\vec{e}=\left(e_{1}, \ldots, e_{r}\right)$ with $2 \leq e_{i} \leq d$ for all $i$, and $2 d-2-g=\sum_{i}\left(e_{i}-1\right)$. Consider the space $M R$ parametrizing tuples consisting of a genus- $g$ curve $C$, points $P_{1}, \ldots, P_{r+3 g}$ on $C$, and a map $f: C \rightarrow \mathbb{P}^{1}$ of degree $d$, ramified to order $e_{i}$ at $P_{i}$ for $i \leq r$ and simply ramified at $P_{r+1}, \ldots, P_{r+3 g}$. Does every component of $M R$ map dominantly to $\mathcal{M}_{g, r}$ under the map induced by forgetting $f$ and $P_{r+1}, \ldots, P_{r+3 g}$ ?

The positive answer to this question in the case $g=0$ is Proposition 3.2. We also remark that Steffen [19] (see also [12]) has a result along these lines for linear series of any degree and dimension, but without any ramification specified. She accomplishes this by studying degeneracy loci of suitable maps of vector bundles; one might try to study our question by looking at Schubert conditions on maps of vector bundles, and suitable intersections of such conditions.

The application of Question 5.4 to irreducibility of Hurwitz spaces is as follows.

THEOREM 5.5. Fix $r, g, d$, and $\vec{e}$ as above. Then a positive answer to Question 5.4 implies that $\mathcal{H}(d, r, g, \vec{e})$ is irreducible, where $\mathcal{H}(d, r, g, \vec{e})$ is the Hurwitz space of covers of $\mathbb{P}^{1}$ of genus $g$ and degree $d$, with a single ramified point of index $e_{i}$ over the ith branch point for $i \leq r$, and simple branching over the remaining branch points. Equivalently, the set of Hurwitz factorizations consisting of $e_{i}$-cycles and $3 g$ transpositions all lie in a single pure braid orbit.

Proof. We first consider the generalization of Proposition 1.1 in this case. The argument for the equivalence of (i) and (ii) goes through unmodified in the generality of higher-genus covers. The argument for the equivalence of (iii) and (iv), where in both cases we prescribe simple ramification at $3 g$ additional unspecified points, is likewise the same as in the genus 0 case. We then have that the Hurwitz space is the image of (a dense open subset of) $M R$, so we see that (iii) or (iv) imply (i) and (ii), and it is enough to check (iv), i.e., to work from the point of view of linear series.

A positive answer to Question 5.4 takes the place of Proposition 3.2, and allows us to work over the generic $r$-marked curve of genus $g$, or more specifically, locally around a given degenerate curve, as in the genus 0 case. Instead of working with a totally degenerate curve, we work with a curve $C_{0}$ consisting of a copy of $\mathbb{P}^{1}$ with $r$ marked points, and with $g$ elliptic tails. As in the proof of [14, Thm. 2.6], the limit linear series on this curve are completely determined by their aspects on $\mathbb{P}^{1}$; on each elliptic tail, they consist of the degree 2 map to $\mathbb{P}^{1}$, simply ramified at the node (and at three other points, which are uniquely determined as 
differing from the node by 2-torsion points). Furthermore, the ramification imposed at each node on $\mathbb{P}^{1}$ is simple ramification; thus, the limit linear series are in natural bijection with the linear series on $\mathbb{P}^{1}$ with the prescribed ramification at $r+g$ points. We know by Theorem 1.2 that the space of these linear series is irreducible as we allow the $r+g$ ramification points to move, so we conclude irreducibility of the space of $\mathfrak{g}_{d}^{1}$,s in a neighborhood of $C_{0}$, and in particular, on the generic $r$-marked curve of genus $g$, as desired.

Results of Conway-Fried-Parker-Völklein (see [9, Appendix], and also [8]) show that, roughly speaking, for any given group and collection of conjugacy classes, if every conjugacy class is repeated often enough, then the components of the Hurwitz space are determined by a certain invariant, called the lifting invariant. Our results fit into the same general philosophy, and might be thought of as an effective version of Conway-Fried-Parker-Völklein for the pure-cycle case.

Finally, we remark that Question 5.4 would potentially have interesting applications to the study of covers in positive characteristic, as well. One cannot hope for a positive answer outside characteristic 0 without some further hypotheses: for instance, in the case $g=0$, the statement is known to fail if one does not require all $e_{i}<p$ (see [16, Ex. 5.6]). However, a positive answer in the case all $e_{i}<p$ would give an important step towards giving new nonexistence results for tame covers in positive characteristic, as is carried out in the genus- 0 case in [17].

We conclude with a brief discussion of the notion of lifting invariants developed by Fried and Serre, including their use by Fried in applying our main results to his theory of modular towers, as well as their likely role in generalizing our results to curves of higher genus. For the sake of simplicity, we restrict our attention to the case of $A_{d}$-covers, and the lifting invariants known as spin invariants, which are discussed in [18] by Serre, who credits Fried for producing an explicit formula, with proofs of some cases. If we have a Hurwitz factorization generating the monodromy group $A_{d}$, the spin invariant takes values in $\{ \pm 1\}$, and, as the name suggests, is an invariant both under simultaneous conjugation, and under the braid action. It can therefore be used to distinguish components of Hurwitz spaces.

However, the spin invariant is an important tool in other contexts as well. For instance, Fried [8] has used our results in the $r=4$ case as a starting point for computations, in which spin invariants play a crucial role, which have allowed him to verify his main conjecture on modular towers in infinite families of examples. The conjecture asserts that there are no rational points at sufficiently high levels of modular towers, which are certain towers of Hurwitz spaces generalizing modular curve towers, and depending on a choice of auxiliary prime $p$. The new families of examples are the first infinite families not arising as modular curve towers for which the conjecture has been verified. Fried had shown earlier [6] that 
in order to verify his conjecture, it suffices to produce at any level of the tower enough $p$-cusps, which are special points on the boundary of the Hurwitz spaces in the tower. In the examples arising from our theorem, there are no $p$-cusps at the bottom level of the tower, but spin invariant computations allow Fried to show that in most cases there are enough $p$-cusps at the next level. Ultimately, he has been able to verify his main conjecture in the genus-0 pure-cycle case (with all cycles odd, so that $G=A_{d}$ ), with $p=2, r=4$ and all four cycles having the same length. For certain $d$, he has also verified the conjecture for all $p$.

Returning to the issue of distinguishing components, spin (and more generally, lifting) invariants are also likely to play an important role in attempts to generalize our understanding of components of Hurwitz spaces. For instance, in [7] Fried studies pure-cycle covers of arbitrary genus, but with all cycles of length 3 , and finds that for positive genus, the Hurwitz space has two components, but they are distinguished by the spin invariant. Thus, the simplest plausible umbrella result for the case of pure-cycle $A_{d}$-covers would be that the components of the Hurwitz space are always determined by their spin invariant. This would not be a complete answer to the question of components, as it would still remain to determine which spin invariants actually occur, but it would nonetheless constitute tremendous progress.

Finally, we would be remiss if we did not mention in this context a closely related question of great importance to applications in inverse Galois theory, and in particular to modular towers: the number of components of a more refined version of the Hurwitz space known as the inner Hurwitz space. In our case that the monodromy group is $A_{d}$, the inner Hurwitz space can have at most two components for each component of the (naive) Hurwitz space, depending on whether or not simultaneous application of an outer automorphism can be achieved via braid transformation. In the examples discussed above, both possibilities occur, so we see that while the components of the inner spaces are not completely determined by the spin invariant, they are determined up to an explicit and frequently tractable factor of at most 2 .

Department of Mathematics, University of California, Davis, One Shields Ave., DAVIS, CA 95616

Department of Mathematics, University of CAlifornia, Berkeley, CA 947203840

REFERENCES

[1] D. Eisenbud and J. Harris, Divisors on general curves and cuspidal rational curves, Invent. Math. 74 (1983), 371-418. 
[2] Limit linear series: Basic theory, Invent. Math. 85 (1986), 337-371.

[3] Irreducibility and monodromy of some families of linear series, Ann. Sci. École Norm. Sup. 20 (1987), no. 1, 65-87.

[4] M. Fried, Fields of definition of function fields and Hurwitz families — groups as Galois groups, Comm. Algebra 5 (1977), no. 1, 17-82.

[5] Relating two genus 0 problems of John Thompson, Progress in Galois Theory, Developments in Mathematics, no. 12, Springer-Verlag, 2005, pp. 51-85.

[6] The Main Conjecture of modular towers and its higher rank generalization, Groupes de Galois arithmetiques et differentiels (Luminy 2004), Seminaires et congres, vol. 13, 2006.

[7] Alternating groups and moduli space lifting invariants, preprint.

[8] Connectedness of families of sphere coverings of a given type, preprint.

[9] M. Fried and H. Völklein, The inverse Galois problem and rational points on moduli spaces, Math. Ann. 290 (1991), no. 4, 771-800.

[10] The embedding problem over a Hilbertian PAC-field, Ann. of Math. 135 (1992), no. 2, 469-481.

[11] W. Fulton, Hurwitz schemes and the irreducibility of moduli of algebraic curves, Ann. of Math. 90 (1969), 542-575.

[12] J. Geertsen, Push-forward of degeneracy classes and ampleness, Proc. Amer. Math. Soc. 129 (2001), no. 7, 1885-1890.

[13] A. Khovanskii and S. Zdravkovska, Branched covers of $s^{2}$ and braid groups, J. Knot Theory Ramifications 5 (1996), no. 1, 55-75.

[14] B. Osserman, The number of linear series on curves with given ramification, Internat. Math. Res. Notices 2003 (2003), no. 47, 2513-2527.

[15] Deformations of covers, Brill-Noether theory, and wild ramification, Math. Res. Lett. 12 (2005), no. 4, 483-491.

[16] Rational functions with given ramification in characteristic p, Compositio Math. 142 (2006), no. 2, 433-450.

[17] Linear series and existence of branched covers, Compositio Math. (to appear).

[18] J. P. Serre, Relèvements dans $\tilde{A}_{n}$, C. R. Acad. Sci. Sér. I. Math. 311 (1990), no. 8, 477-482.

[19] F. Steffen, A generalized principal ideal theorem with an application to Brill-Noether theory, Invent. Math. 132 (1998), no. 1, 73-89.

[20] H. Völklein, Groups as Galois Groups, Cambridge Stud. Adv. Math., no. 53, Cambridge University Press, 1996.

[21] A. Williamson, On primitive permutation groups containing a cycle, Math. Z. 130 (1973), $159-162$. 